\title{
ANKDD1A may serve as a critical gene in the immune microenvironment of breast cancer
}

\author{
Ping Zhao ${ }^{1 \#}$, Yang Fu ${ }^{2 \#}$, Xin Yang ${ }^{3}$, Hiseein Ali Abdoulaye ${ }^{4}$, Ritika Rauniyar ${ }^{4}$, Jing Peng ${ }^{5}$, Maohua Wang ${ }^{1}$, \\ Huiqiao Wang ${ }^{4}$, Minjie $\mathrm{Ning}^{4}$, Ying Chen ${ }^{4} \wedge$, Yunchao Huang ${ }^{4}$
}

${ }^{1}$ Department of Mammary Surgery I, The Third Affiliated Hospital of Kunming Medical University (Yunnan Cancer Hospital, Yunnan Cancer Center), Kunming, China; ${ }^{2}$ Department of Radiology, The First People's Hospital of Kunming, Kunming, China; ${ }^{3}$ Department of Blood Transfusion, The First People's Hospital of Yunnan Province, The Affiliated Hospital of Kunming University of Science and Technology, Kunming, China; ${ }^{4}$ Department of Thoracic Surgery I, The Third Affiliated Hospital of Kunming Medical University (Yunnan Cancer Hospital, Yunnan Cancer Center), The International Cooperation Key Laboratory of Regional Tumor in High Altitude Area, Kunming, China; ${ }^{5}$ Anesthesiology Department, The Third Affiliated Hospital of Kunming Medical University (Yunnan Cancer Hospital, Yunnan Cancer Center), The International Cooperation Key Laboratory of Regional Tumor in High Altitude Area, Kunming, China

Contributions: (I) Conception and design: P Zhao, Y Fu, Y Chen, Y Huang; (II) Administrative support: Y Chen, Y Huang; (III) Provision of study materials or patients: X Yang, HA Abdoulaye, R Rauniyar, J Peng; (IV) Collection and assembly of data: M Wang, H Wang, M Ning; (V) Data analysis and interpretation: P Zhao, Y Fu, Y Chen, Y Huang, J Peng; (VI) Manuscript writing: All authors; (VII) Final approval of manuscript: All authors.

"These authors contributed equally to this work.

Correspondence to: Ying Chen. Department of Thoracic Surgery, The Third Affiliated Hospital of Kunming Medical University (Yunnan Cancer Hospital, Yunnan Cancer Center), No. 519, Kunzhou Road, Kunming 650118, China. Email: 36410008@qq.com; Yunchao Huang. The Third Affiliated Hospital of Kunming Medical University (Yunnan Cancer Hospital, Yunnan Cancer Center), No. 519, Kunzhou Road, Kunming 650118, China. Email: huangych2001@aliyun.com.

Background: Breast cancer (BC), a very heterogeneous systemic disease, is the most frequently seen malignancy in women, especially in some developed countries or regions. Estrogen receptor (ER), progesterone receptor (PR), human epidermal growth factor receptor 2 (HER2) and Ki67 have been extensively used to predict the clinical outcome, but it is still a hotspot to search for more predictive prognostic markers. Ankyrin repeat and death domain containing 1A (ANKDD1A), which contains nine ankyrin repeats, has been discovered to play a role of tumor suppressor in glioblastoma multiforme (GBM). However, its role in BC remains unknown so far.

Methods: ANKDD1A expression and clinical information of BC were extracted from the TCGA dataset. Then, the ANKDD1A expression level was explored in BC from different perspectives, including clinical stage, molecular subtype, histology type and immune microenvironment. Afterwards, functional enrichment analysis of ANKDD1A co-expressed genes was carried out to estimate the role of ANKDD1A in BC, and the methylation status of ANKDD1A was evaluated by MEXPRESS. In addition, the correlation of ANKDD1A with immunocytes was explored, and survival analysis was carried out to evaluate the prognostic value of ANKDD1A in BC.

Results: ANKDD1A decreased in BC compared with the para-cancerous tissues. Additionally, ANKDD1A was up-regulated in early-stage BC, ER negative group, infiltrating lobular carcinoma, and the normal subtype in BC molecular subtypes. According to functional enrichment analysis, ANKDD1A co-expressed genes were mainly involved in the immune process. Also, our results revealed that ANKDD1A was tightly associated with T cells. Survival analysis suggested that, patients with higher ANKDD1A expression had more favorable prognosis than those with lower ANKDD1A expression.

^ ORCID: 0000-0003-0582-380X. 
Conclusions: ANKDD1A may serve as a critical gene in the pathogenesis of $\mathrm{BC}$ and the immune microenvironment of BC tissues.

Keywords: Breast cancer (BC); ankyrin repeat and death domain containing 1A (ANKDD1A); tumor microenvironment; immune; prognosis

Submitted Aug 07, 2020. Accepted for publication Jan 22, 2021.

doi: $10.21037 /$ tcr-20-2685

View this article at: http://dx.doi.org/10.21037/tcr-20-2685

\section{Introduction}

Breast cancer (BC) is an extremely heterogeneous systemic disease and the second leading cause of death worldwide (1). There are over 20 pathological types of invasive $\mathrm{BC}$, which exhibit different prognostic outcomes. At present, the expression levels of estrogen receptor (ER), progesterone receptor (PR), human epidermal growth factor receptor 2 (HER2) and Ki67 have been adopted as important reference to select clinical treatments (2), but the individualized treatment for $\mathrm{BC}$ cannot be reached so far. Therefore, it is still a hot topic in BC research to identify more predictive prognostic markers.

Ankyrin repeat and death domain containing 1A (ANKDD1A), is located at $15 \mathrm{q} 22.31$, and contains nine ankyrin repeats and one death domain. Meanwhile, it is down-regulated in glioblastoma multiforme (GBM), and acts as a tumor suppressor in GBM, especially under hypoxic condition (3). Importantly, the ankyrin repeat, one of the most common protein-protein interaction (PPI) motifs, has been reported to be involved in numerous functions, such as transcriptional activator, transporters, inflammatory responses, and signal transducers (4). Nonetheless, the role of ANKDD1A in BC remains unclear for the time being.

In this study, we discussed ANKDD1A expression in $\mathrm{BC}$, including the correlation of ANKDD1A with various clinical phenotypes (like clinical stage and molecular subtypes). According to the functional enrichment analysis of ANKDD1A-related genes, ANKDD1A mostly participated in immune response. Moreover, ANKDD1A was shown to be strongly related to TBX21 and STAT4, the makers of Th1 cells. Most importantly, patients with higher ANKDD1A expression had superior clinical outcomes.

We present the following article in accordance with the MDAR checklist (available at http://dx.doi.org/10.21037/ tcr-20-2685).

\section{Methods}

\section{Data sources}

In this study, the clinical information of BC and ANKDD1A expression profile were obtained from The Cancer Genome Atlas (TCGA) database (https://portal.gdc.cancer.gov/) (5). The dataset of TCGA-BC contained 113 Para-cancerous breast tissues and 1,109 BC tissues. Specifically, clinical information was collected from BC individuals, including clinical stage, T stage, ER status, molecular subtypes and pathological types, while ANKDD1A expression was extracted from the RNA-sequencing data. Overall survival (OS) was calculated as the duration from the date of diagnosis to the date of death or last follow-up. In addition, the immune scores of cancer samples were obtained from the ESTIMATE (https://bioinformatics.mdanderson. org/estimate/), the professional approach to estimate the immune and stromal scores of tumor samples (6). The study was conducted in accordance with the Declaration of Helsinki (as revised in 2013).

\section{ANKDD1A expression}

ANKDD1A expression in para-cancerous breast tissues and $\mathrm{BC}$ tissues was analyzed, which was also estimated among BC individuals with different clinical stages, $\mathrm{T}$ stages, ER statuses, molecular subtypes, and pathological types.

\section{Gene functional enrichment analysis of ANKDD1A}

To explore the molecular function (MF) of ANKDD1A, the ANKDD1A co-expressed genes that had the absolute value of Spearman's correlation coefficient of $>0.4$ were selected. Thereafter, functional enrichment analysis was carried out by the Cluster Profiler package provided by $\mathrm{Yu}$ et al. (7). 

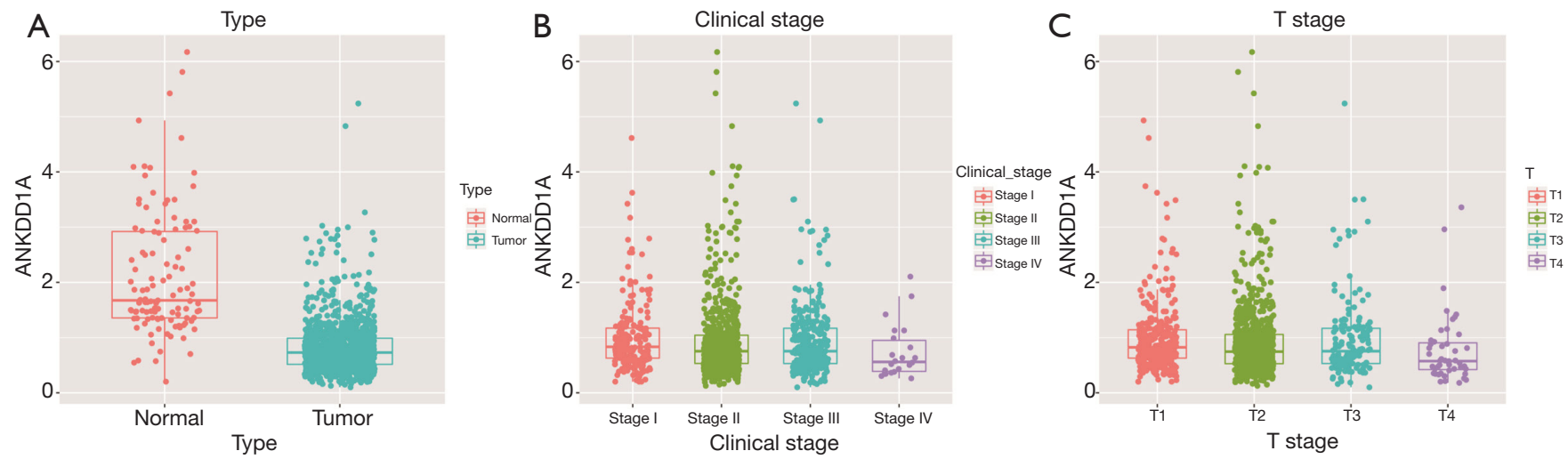

Figure 1 ANKDD1A expression in BC. (A) ANKDD1A is down-regulated in breast cancer tissues compared to Para-cancerous tissues. (B) ANKDD1A has a downtrend while the clinical staging of tumor progressed. (C) ANKDD1A has a decrease with T stages in the progression of breast cancer. ANKDD1A, ankyrin repeat and death domain containing 1A.

\section{Immune-related analysis}

Individuals were divided into high or low immunescore group according to the median immune score of samples. Later, ANKDD1A expression in these two groups was analyzed. The correlations of ANKDD1A with immunocytes (including B cells, $\mathrm{CD}^{+} \mathrm{T}$ cells, $\mathrm{CD} 4^{+} \mathrm{T}$ cells, macrophages, neutrophils, and dendritic cells) were evaluated by TIMER (https://cistrome.shinyapps.io/timer) (8). In addition, the relationships of ANKDD1A with various immunocyte markers were estimated by TIMER.

\section{Survival analysis}

The Kaplan-Meier plot analysis was adopted in estimating the OS for BC individuals using log-rank test.

\section{Methylation status of ANKDD1A}

The methylation status of ANKDD1A in BC was evaluated by the MEXPRESS website (https://mexpress.be/).

\section{Statistical analysis}

The SPSS23.0 software was utilized in all statistical analyses, and Fisher exact test was adopted to analyze the categorical data. A two-sided $\mathrm{P}$ value of 0.05 indicated statistically significant difference.

\section{Results}

Down-regulation of ANKDD1A in BC

Firstly, ANKDD1A expression was compared between $\mathrm{BC}$ tissues and para-cancerous breast tissues. As shown in Figure $1 A$, ANKDD1A was significantly down-regulated in cancer tissues. Later, ANKDD1A expression was evaluated at different clinical stage, which suggested that ANKDD1A showed a downtrend as the tumor clinical stage progressed (Figure $1 B$ ). Also, ANKDD1A was down-regulated as T stage of BC progressed (Figure 1C).

\section{Relationship of ANKDD1A to the molecular subtype of BC}

Firstly, ANKDD1A expression in different histological subtypes was evaluated, which revealed that ANKDD1A was up-regulated in infiltrating lobular carcinoma compared with that in infiltrating ductal carcinoma (Figure $2 A$ ). As a supplement to pathological type, ANKDD1A expression in different molecular types was analyzed based on the gene expression profiles and molecular biological characteristics of individuals. According to our results, ANKDD1A showed the highest level in normal type, followed by basal type, luminal A, and luminal B, while the lowest level was detected in HER2-enriched type of BC (Figure 2B). Additionally, it was discovered that, ANKDD1A was upregulated in ER negative group compared with that in ER positive group (Figure 2C). 

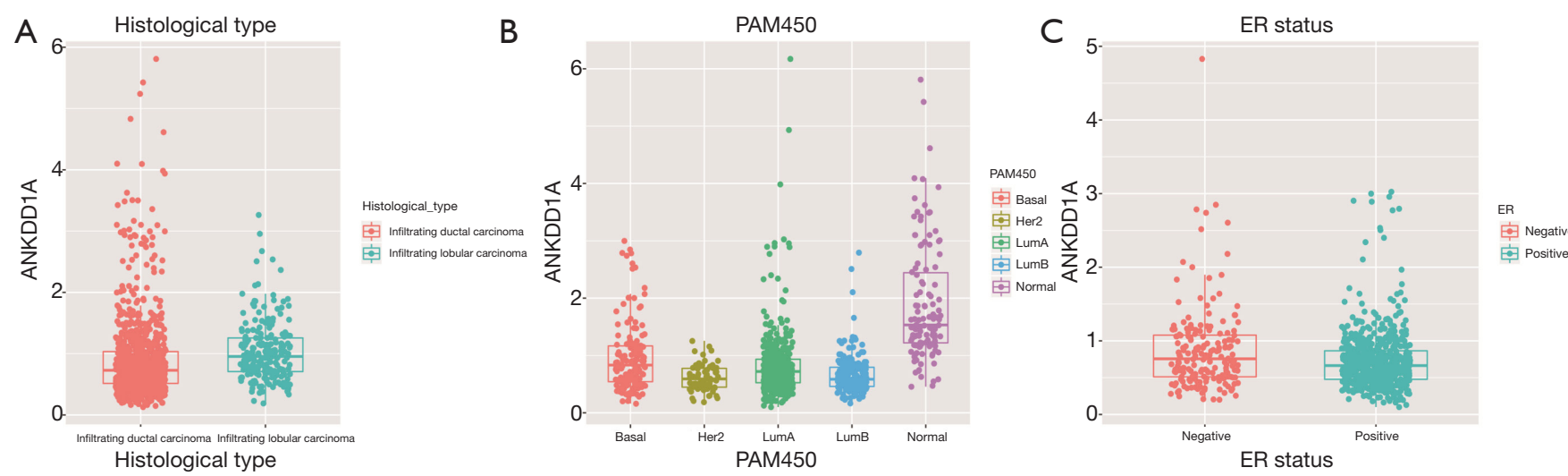

亩 Positive

Figure 2 ANKDD1A expression in different subgroup. (A) ANKDD1A has a higher expression in infiltrating lobular carcinoma compared to infiltrating ductal carcinoma. (B) ANKDD1A has a highest level in normal type, followed by basal type, luminal A, and luminal B, has a lowest level in HER2-enriched type of breast cancer. (C) ANKDD1A has a higher expression in ER negative group compared to the ER positive group. ANKDD1A, ankyrin repeat and death domain containing 1A; HER2, human epidermal growth factor receptor 2; ER, estrogen receptor.
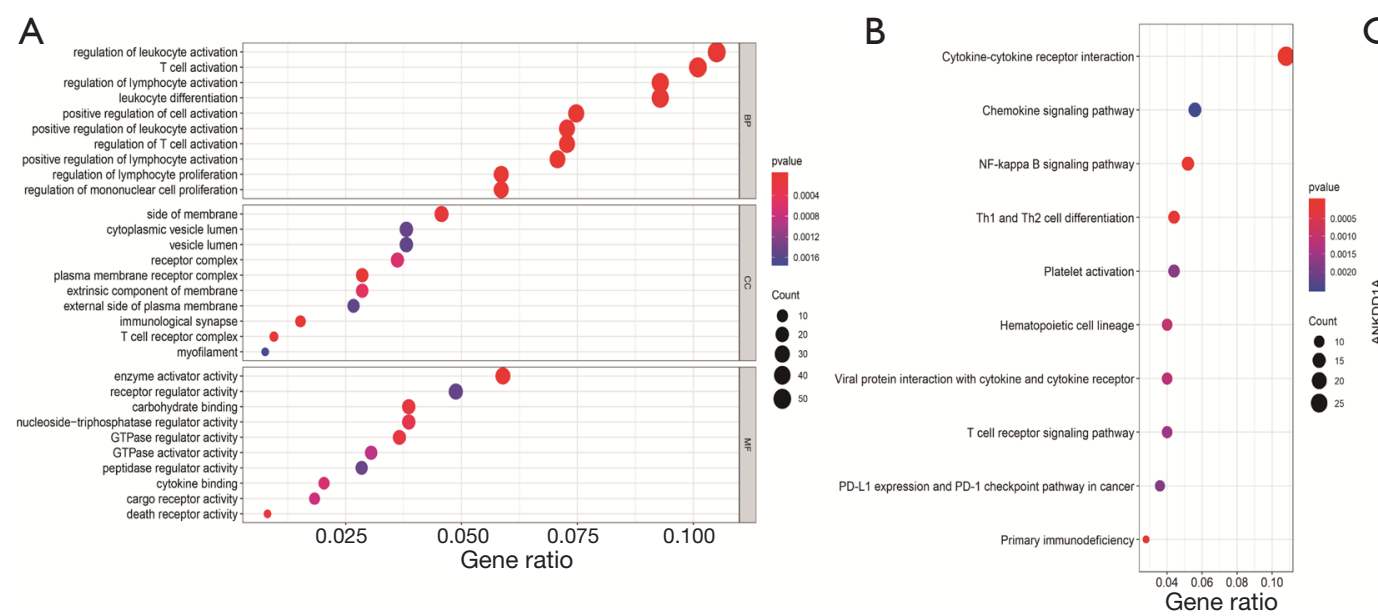

C

Figure 3 ANKDD1A involved in immune process. (A) GO analysis of ANKDD1A related co-expressed genes. (B) KEGG analysis of ANKDD1A related co-expressed genes. (C) ANKDD1A has a higher expression level in high-immune score group compared to lowimmune score group. ANKDD1A, ankyrin repeat and death domain containing 1A; GO, Gene Ontology; KEGG, Kyoto Encyclopedia of Genes and Genomes.

\section{ANKDD1A and immune process}

To investigate the gene function of ANKDD1A in BC, genes with high co-expression with ANKDD1A in BC were selected. A total of 633 co-expressed genes were identified according to the thresholds of $\mathrm{P}$ value $<0.05$ and $\mid$ coefficient $\mid \geq 0.4$. Later, gene functional enrichment analysis was carried out, including biological MF, biological process (BP) and cellular component (CC) (Figure 3A). Interestingly, in $\mathrm{BP}$ analysis, the ANKDD1A coexpressed genes were mainly involved in the immune process, such as regulation of leukocyte activation, $\mathrm{T}$ cell activation, regulation of lymphocyte activation, leukocyte differentiation, positive regulation of leukocyte activation, and regulation of $\mathrm{T}$ cell activation. As for $\mathrm{CC}$ analysis, the ANKDD1A co-expressed genes were mainly enriched in immunological synapse and $\mathrm{T}$ cell receptor complex. With regard to Kyoto Encyclopedia of Genes and Genomes (KEGG) analysis (Figure 3B), the ANKDD1A co-expressed genes were highly enriched in the immune-related signaling pathways, including Th1 and Th2 cell differentiation, 


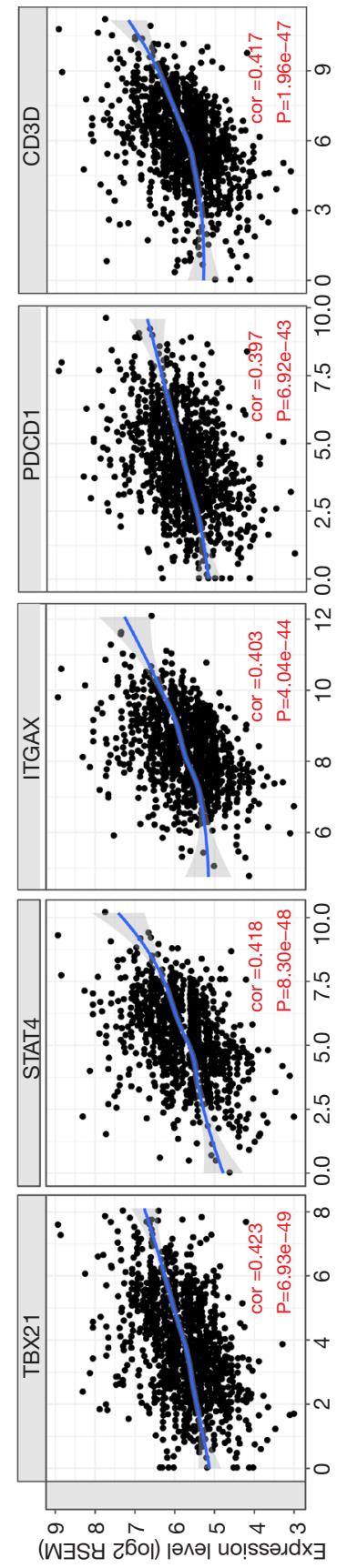

$\infty$

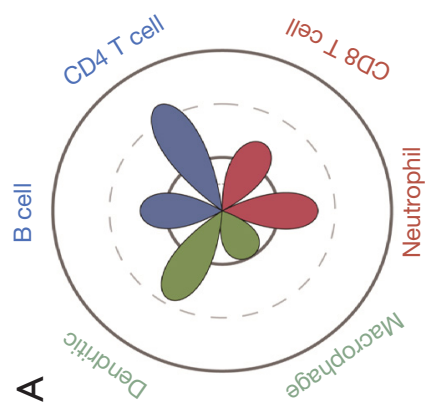

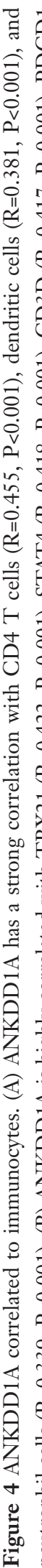

Primary immunodeficiency, $\mathrm{T}$ cell receptor signaling pathway, PD-L1 expression, and PD-1 checkpoint pathway in cancer. Taken together, ANKDD1A played a vital role in immune response, especially in $\mathrm{T}$ cells.

$\mathrm{BC}$ individuals were divided into two groups according to the median immune score, and ANKDD1A expression was compared between these two groups. As displayed in Figure 3C, patients with higher immune scores had high ANKDD1A expression.

\section{ANKDD1A correlation with T cells}

Functional enrichment analysis of ANKDD1A co-expressed genes revealed that, these genes were mainly enriched in the immune process, Therefore, the correlations of ANKDD1A with immunocytes were analyzed. As exhibited in Figure 4A, ANKDD1A was highly correlated with CD4 T cells ( $\mathrm{R}=0.455, \mathrm{P}<0.001)$, dendritic cells $(\mathrm{R}=0.381, \mathrm{P}<0.001)$, and neutrophils $(\mathrm{R}=0.339, \mathrm{P}<0.001)$. Afterwards, the relationships of ANKDD1A with immunocyte markers were analyzed. According to Table 1, ANKDD1A was highly correlated with TBX21 ( $\mathrm{R}=0.422, \mathrm{P}<0.001)$, STAT4 $(\mathrm{R}=0.418$, $\mathrm{P}<0.001)$, CD3D $(\mathrm{R}=0.417, \mathrm{P}<0.001)$, PDCD1 $(\mathrm{R}=0.400$, $\mathrm{P}<0.001)$, and ITGAX $(\mathrm{R}=0.400, \mathrm{P}<0.001)$ (Figure $4 B)$. Among these markers, TBX21, STAT3, and PDCD1 were the markers indicating exhaustion of Th1 cells and T cells, consistent with our previous results.

\section{ANKDD1A was a marker that predicted superior prognosis for $B C$}

ANKDD1A might be involved in immune response, and it was a critical factor for the clinical outcome of BC. Thus, we evaluated the prognostic value of ANKDD1A in BC. It was observed in Figure 5 that, patients with higher ANKDD1A expression achieved a longer survival.

\section{Methylation status of ANKDD1A in BC}

As suggested in previous study, ANKDD1A is hypermethylated in GBM. As a result, we explored the ANKDD1A methylation status in BC using the online tools. According to Figure 6, ANKDD1A expression was correlated with different $\mathrm{CpG}$ islands, including cg18612204, cg10097464, cg00958451, cg21153004, cg12119988, and cg09291474. SH3BP2/HIF 1 AN interacts with $A N K D D 1 A$ gene, and the correlation between ANKDD1A and SH3BP2/HIF1AN is 0.296 
Table 1 The correlation between ANKDD1A and immune cell markers

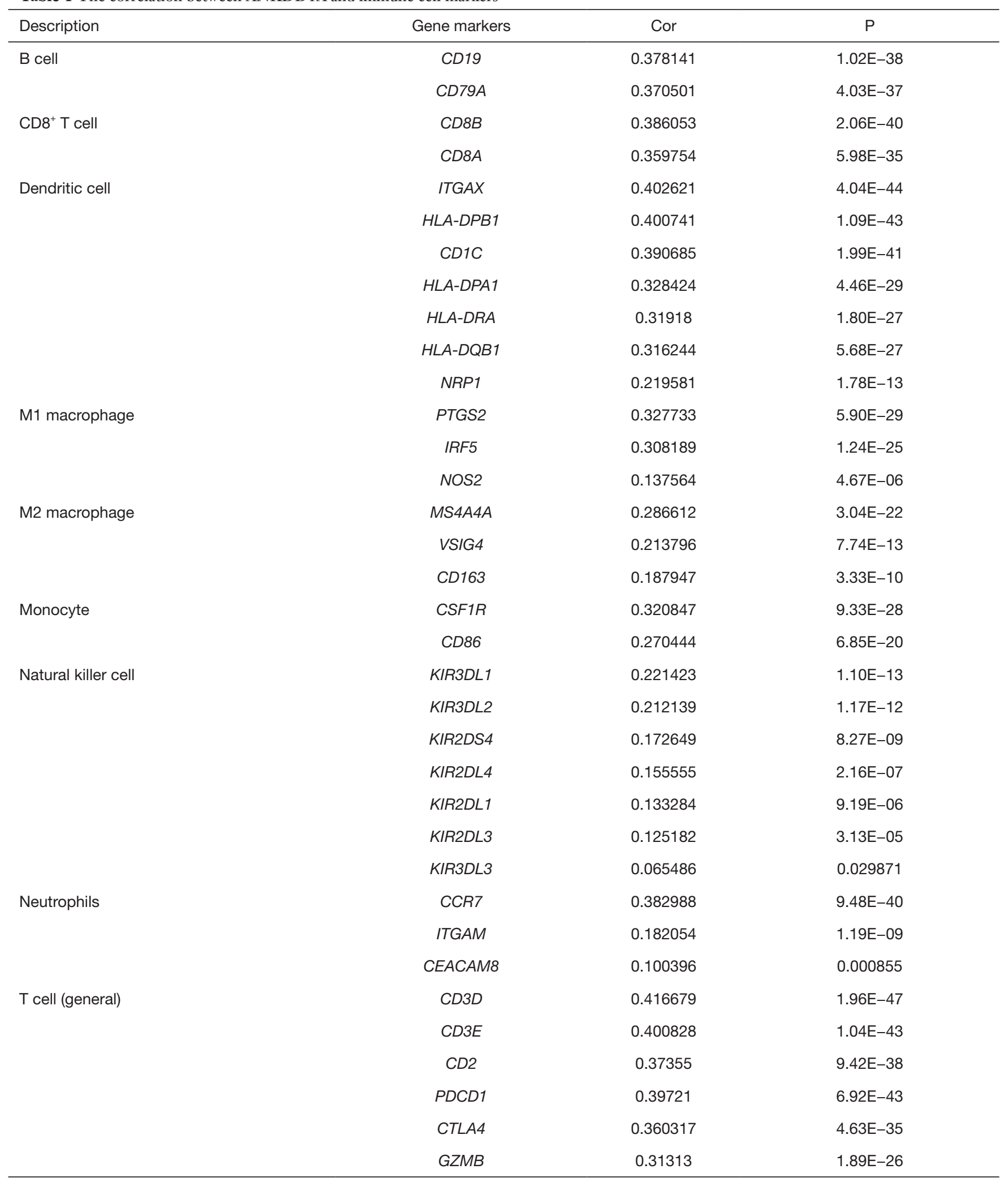

Table 1 (continued) 
Table 1 (continued)

\begin{tabular}{|c|c|c|c|}
\hline Description & Gene markers & Cor & $\mathrm{P}$ \\
\hline & LAG3 & 0.308372 & $1.16 \mathrm{E}-25$ \\
\hline & HAVCR2 & 0.244688 & $1.85 \mathrm{E}-16$ \\
\hline \multirow[t]{3}{*}{ TAM } & CCL2 & 0.371405 & $2.62 \mathrm{E}-37$ \\
\hline & IL10 & 0.255757 & $6.90 \mathrm{E}-18$ \\
\hline & $C D 68$ & 0.222247 & 8.91E-14 \\
\hline \multirow[t]{2}{*}{ Tfh } & BCL6 & 0.211027 & $1.54 \mathrm{E}-12$ \\
\hline & IL21 & 0.161266 & $7.54 \mathrm{E}-08$ \\
\hline \multirow[t]{5}{*}{ Th1 } & TBX21 & 0.422625 & $6.93 \mathrm{E}-49$ \\
\hline & STAT4 & 0.418217 & $8.30 \mathrm{E}-48$ \\
\hline & IFNG & 0.246152 & $1.21 \mathrm{E}-16$ \\
\hline & TNF & 0.224138 & $5.42 \mathrm{E}-14$ \\
\hline & STAT1 & 0.004721 & 0.875713 \\
\hline \multirow[t]{2}{*}{ Th17 } & IL17A & 0.097579 & 0.001194 \\
\hline & STAT3 & -0.08253 & 0.006168 \\
\hline \multirow[t]{4}{*}{ Th2 } & STAT5A & 0.297464 & $6.52 \mathrm{E}-24$ \\
\hline & IL13 & 0.280285 & 2.64E-21 \\
\hline & STAT6 & 0.159128 & $1.12 \mathrm{E}-07$ \\
\hline & GATA3 & -0.27495 & 1.57E-20 \\
\hline \multirow[t]{4}{*}{ Treg } & TGFB1 & 0.350518 & $3.79 \mathrm{E}-33$ \\
\hline & FOXP3 & 0.271139 & $5.46 \mathrm{E}-20$ \\
\hline & STAT5B & 0.138381 & $4.09 \mathrm{E}-06$ \\
\hline & CCR8 & 0.117085 & 9.93E-05 \\
\hline
\end{tabular}

ANKDD1A, ankyrin repeat and death domain containing $1 \mathrm{~A}$.

and 0.102, respectively (Table 2). As showed in Figure 6, ANKDD1A is regulated by methylation, so the expression of ANKDD1A in BC is inhibited. And, SH3BP2/HIF1AN interacts with $A N K D D 1 A$ gene to inhibit tumor immune microenvironment, thus promoting tumor growth.

\section{Discussion}

Findings in this study suggested that, ANKDD1A decreased in BC tissues compared with para-carcinoma tissues. Besides, high ANKDD1A expression was detected in earlystage BC, ER negative group, infiltrating lobular carcinoma, and normal subtype of BC molecular subtypes. Noticeably, ANKDD1A expression was correlated with several CpG islands, such as cg18612204, cg10097464, cg00958451, cg21153004, cg12119988 and cg09291474.

DNA methylation is a common and important form of epigenetics, which plays an important role in the occurrence and development of malignant tumors. Methylation regulation is quite common in $\mathrm{BC}$, which involves a wide range of genes, including cell cycle regulatory genes, DNA repair genes, signal transduction genes, and cell adhesion genes. Typically, the abnormal DNA methylation may serve as a potential biomarker in the early diagnosis of BC. In this study, we found that ANKDD1A expression was related to its CpG islands, indicating that the methylation status of ANKDD1A might substantially contribute to BC. However, the precise mechanism in the regulation of ANKDD1A methylation remains unknown so far, which deserves further investigation. 
In addition, gene functional enrichment analysis indicated that, ANKDD1A co-expressed genes were mainly involved in the immune process. Therefore, the correlations of ANKDD1A with immunocytes were evaluated. The results suggested that, ANKDD1A was positively correlated with CD4 cells. Furthermore, the correlations between

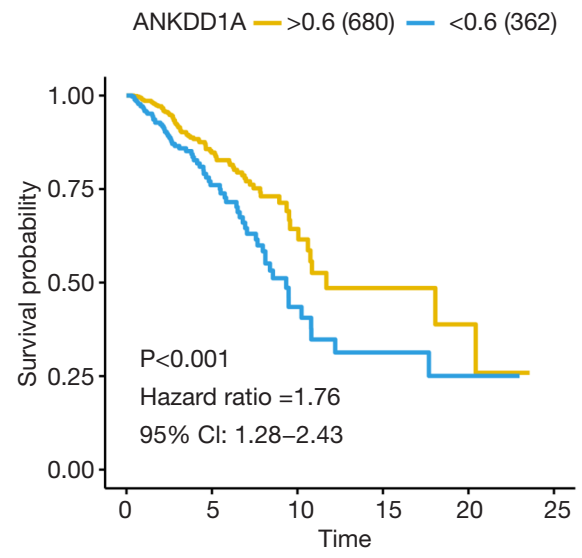

Figure 5 ANKDD1A prognostic value in BC. Patient with higher expression of ANKDD1A has a promising prognosis compared to those with lower expression of ANKDD1A. ANKDD1A, ankyrin repeat and death domain containing $1 \mathrm{~A}$.
ANKDD1A and immunocyte markers revealed that, ANKDD1A was closely related to TBX21, STAT3 and PDCD1, the markers of $T$ cells. These results indicated that, ANKDD1A was strongly associated with the immune microenvironment of BC.

Mina Bissel first proposed that, the biological behavior of malignant tumors depended not only on tumor cells, but also on the tumor microenvironment (9), and it also exerted a decisive role in tumor evolution and prognosis, as proved by numerous studies (10-12). The microenvironment of BC cells consists of cancer cells, fibroblasts, endothelial cells, immune cells, adipocytes and other stromal cells, as well as the extracellular matrix components, which jointly promote the occurrence and development of BC (13).

$\mathrm{T}$ lymphocytes are the major immune infiltrating cells in $\mathrm{BC}$, which account for approximately $75 \%$, while $\mathrm{B}$ lymphocytes take up less than $20 \%$, and monocytes occupy $10 \%$ (14). T lymphocytes can be divided into CD4 T cells, CD8 T cells and Treg cells (15). According to the secreted cytokines, $\mathrm{CD}^{+} \mathrm{T}$ cells are classified as Th1 and Th2 cells. Of them, Th1 cells enhance cellular immunity through secreting IFN- $\gamma$ and IL-2 (16), while Th2 cells inhibit cellular immunity and promote humoral immunity by secreting IL-4, IL-5 and IL-10 $(17,18)$.

The potent correlation between ANKDD1A and T cells
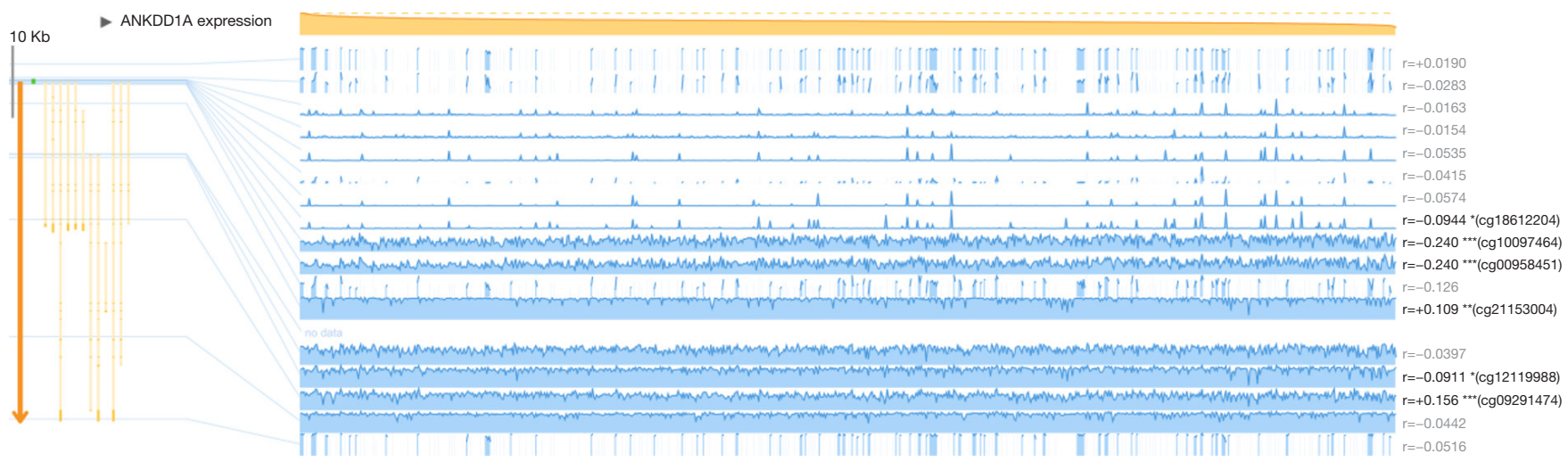

Figure 6 ANKDD1A expression is correlated with the methylation of its CpG islands. *, $\mathrm{P}<0.05 ;{ }^{* *}, \mathrm{P}<0.01 ;{ }^{* * *}$, $\mathrm{P}<0.001$. ANKDD1A, ankyrin repeat and death domain containing $1 \mathrm{~A}$.

Table 2 The correlation between ANKDD1A and regulatory genes

\begin{tabular}{lccc}
\hline Description & Gene markers & Cor & P \\
\hline Regulatory genes & SH3BP2 & 0.296587073 & $9.38 \mathrm{E}-24$ \\
Regulatory genes & HIF1AN & 0.102758753 & 0.000645518 \\
\hline
\end{tabular}


suggests that, ANKDD1A contributes to the regulation of $\mathrm{T}$ cells, especially for Th1 cells. Also, studies reveal that the increased proportion of Th1 cells and decreased proportion of Th2 cells indicate favorable prognosis for $\mathrm{BC}$; in addition, the ratio of Th1/Th2 in BC tissues has become one of the optimal immune prognostic factors (19). Further, survival analysis of ANKDD1A showed that, individuals with high ANKDD1A expression had promising clinical outcomes compared with those with low ANKDD1A expression. Such result was consistent with the finding that, BC patients with a higher Th1 cell proportion had better prognosis. In future, we will perform experimental studies to explore the function of ANKDD1A in BC, so as to verify our results.

\section{Conclusions}

ANKDD1A may serve as a critical gene in the pathogenesis of $\mathrm{BC}$ and the immune microenvironment of $\mathrm{BC}$ tissues.

\section{Acknowledgments}

Funding: This work was supported by National Natural Science Foundation of China (81702274); National Key Research and Development Projects (2017YFC0907902), Natural Science Foundation of Yunnan Province (2017FE468-214, 2017FE468-159); Yunnan Health Training Project of High Level Talents (H-2018025).

\section{Footnote}

Reporting Checklist: The authors have completed the MDAR reporting checklist. Available at http://dx.doi.org/10.21037/ tcr-20-2685

Conflicts of Interest: All authors have completed the ICMJE uniform disclosure form (available at http://dx.doi. org/10.21037/tcr-20-2685). The authors have no conflicts of interest to declare.

Ethical Statement: The authors are accountable for all aspects of the work in ensuring that questions related to the accuracy or integrity of any part of the work are appropriately investigated and resolved. The study was conducted in accordance with the Declaration of Helsinki (as revised in 2013).

Open Access Statement: This is an Open Access article distributed in accordance with the Creative Commons Attribution-NonCommercial-NoDerivs 4.0 International License (CC BY-NC-ND 4.0), which permits the noncommercial replication and distribution of the article with the strict proviso that no changes or edits are made and the original work is properly cited (including links to both the formal publication through the relevant DOI and the license). See: https://creativecommons.org/licenses/by-nc-nd/4.0/.

\section{References}

1. Siegel RL, Miller KD, Jemal A. Cancer statistics, 2019. CA Cancer J Clin 2019;69:7-34.

2. Joensuu K, Leidenius M, Kero M, et al. ER, PR, HER2, Ki-67 and CK5 in Early and Late Relapsing Breast Cancer-Reduced CK5 Expression in Metastases. Breast Cancer (Auckl) 2013;7:23-34.

3. Feng J, Zhang Y, She X, et al. Hypermethylated gene ANKDD1A is a candidate tumor suppressor that interacts with FIH1 and decreases HIF1 $\alpha$ stability to inhibit cell autophagy in the glioblastoma multiforme hypoxia microenvironment. Oncogene 2019;38:103-19.

4. Wolf D, Hofbrucker-MacKenzie SA, Izadi M, et al. Ankyrin repeat-containing N-Ank proteins shape cellular membranes. Nat Cell Biol 2019;21:1191-205.

5. Tomczak K, Czerwińska P, Wiznerowicz M. The Cancer Genome Atlas (TCGA): an immeasurable source of knowledge. Contemp Oncol (Pozn) 2015;19:A68-77.

6. Yoshihara K, Shahmoradgoli M, Martínez E, et al. Inferring tumour purity and stromal and immune cell admixture from expression data. Nat Commun 2013;4:2612.

7. Yu G, Wang LG, Han Y, et al. clusterProfiler: an R package for comparing biological themes among gene clusters. OMICS 2012;16:284-7.

8. Li T, Fan J, Wang B, et al. TIMER: A Web Server for Comprehensive Analysis of Tumor-Infiltrating Immune Cells. Cancer Res 2017;77:e108-10.

9. Bissell MJ, Hines WC. Why don't we get more cancer? A proposed role of the microenvironment in restraining cancer progression. Nat Med 2011;17:320-9.

10. Wang $M$, Zhao J, Zhang L, et al. Role of tumor microenvironment in tumorigenesis. J Cancer 2017;8:761-73.

11. Ariztia EV, Lee CJ, Gogoi R, et al. The tumor microenvironment: key to early detection. Crit Rev Clin Lab Sci 2006;43:393-425.

12. Yuan Y, Jiang YC, Sun CK, et al. Role of the tumor microenvironment in tumor progression and the clinical 
applications (Review). Oncol Rep 2016;35:2499-515.

13. Eftekhari R, Esmaeili R, Mirzaei R, et al. Study of the tumor microenvironment during breast cancer progression. Cancer Cell Int 2017;17:123.

14. Ahn SG, Jeong J, Hong S, et al. Current Issues and Clinical Evidence in Tumor-Infiltrating Lymphocytes in Breast Cancer. J Pathol Transl Med 2015;49:355-63.

15. Carvalho MI, Pires I, Prada J, et al. A role for T-lymphocytes in human breast cancer and in canine mammary tumors. Biomed Res Int 2014;2014:130894.

16. Viallard JF, Pellegrin JL, Ranchin V, et al. Th1 (IL-2, interferon-gamma (IFN-gamma)) and Th2 (IL-10, IL-4) cytokine production by peripheral blood mononuclear cells
(PBMC) from patients with systemic lupus erythematosus (SLE). Clin Exp Immunol 1999;115:189-95.

17. Jacysyn JF, Abrahamsohn IA, Macedo MS. IL-4 from Th2-type cells suppresses induction of delayed-type hypersensitivity elicited shortly after immunization. Immunol Cell Biol 2003;81:424-30.

18. van Oosterhout AJ, Motta AC. Th1/Th2 paradigm: not seeing the forest for the trees? Eur Respir J 2005;25:591-3.

19. DeNardo DG, Coussens LM. Inflammation and breast cancer. Balancing immune response: crosstalk between adaptive and innate immune cells during breast cancer progression. Breast Cancer Res 2007;9:212.

Cite this article as: Zhao P, Fu Y, Yang X, Abdoulaye HA, Rauniyar R, Peng J, Wang M, Wang H, Ning M, Chen Y, Huang Y. ANKDD1A may serve as a critical gene in the immune microenvironment of breast cancer. Transl Cancer Res 2021;10(3):1358-1367. doi: 10.21037/tcr-20-2685 